\title{
The location of T1 diahetes associated SNPs in regulatory regions
}

\section{S. Beka', I. te Boekhorst ${ }^{1 \bowtie}$, I. Abnizova}

'School of Computer Science, University of Hertfordshire, Hatfield, United Kingdom

${ }^{2}$ Wellcome Trust Sanger Institute, Hinxton, United Kingdom

\section{Motivations}

Although many association studies on complex diseases focus on variation in coding DNA, recent research shows increasing evidence that the cause of such diseases should be sought in the regulation of gene activity. Rather than studying mutations in genes coding for transcription factors, my work focuses on genetic variants (SNPS) in regulatory modules (TFBS, enhancers, promoters, or other genic locations likely to be involved in gene regulation such as UTR, introns and splice junctions) that are in Type 1 Diabetes (TID) susceptibility regions of the human genome. The specific research question is: are SNPS associated with TID more likely to occur in (putative) regulatory regions than other SNPs found in TID susceptible regions? In addition: Because genes may overlap and/or occurrence in multiple transcripts, one and the same SNP may be associated with more than one genic location and affect more than one functional region (e.g. is a mutation in as well a coding region as a regulatory region). Are SNPS associated with a complex disease such as TID more likely to be of this kind?

\section{Methods}

An extensive search in the databases ENSEMBL and TIDbase was conducted to collect information all SNPs in TID susceptible regions [Coordinates on genome, type of variant (mutant allele, wildtype allele, transversion or transition, synonymous or non-synonymous), transcriptID, intra-genic region (up/downstream, exon, intron, splice junction, UTR, type of (micro)RNA), type of gene (coding/pseudo/microRNA) and associated diseases] A statistical analysis was performed to assess possible associations between the status of a SNP (associated/not-associated with TID) on the one hand and features characterising the (intra/inter-) genic position on the other hand.

\section{Results}

1) SNPs associated with TID are more likely to occur in regulatory regions than those that are not associated with TID 2) SNPs associated with TID occur more often in multiple genic locations than those that occur in only one genic location. They appear to be relatively over-abundant in transcription factor binding sites, introns and splice-sites. 